\title{
INSATISFAÇÃO COM O PESO E A MASSA \\ CORPORAL EM ESTUDANTES DO \\ ENSINO FUNDAMENTAL E MÉDIO DO \\ SEXO FEMININO NO MUNICÍPIO DO \\ RIO DE JANEIRO
}

\section{DR. ALEXANDRE PALMA}

Doutor em Saúde Pública pela Escola Nacional de Saúde Pública da Fundação Oswaldo Cruz (ENSP/FIOCRUZ), Professor Adjunto da Escola de Educação Física e Desportos e do Programa de Pós-graduação em Educação Física da Universidade Federal do Rio de Janeiro (PPGEF/EEFD/NFRJ)

(Rio de Janeiro - Rio de Janeiro - Brasil)

E-mail: palma_alexandre@yahoo.com.br

\section{MS. FABIANA RESENDE}

Mestre em Educação Física pelo Programa de Pós-graduação em Educação Física da Universidade Federal do Rio de Janeiro (PPGEF/EEFD/UFRJ) (Rio de Janeiro - Rio de Janeiro - Brasil)

E-mail: fabianardoespirito@yahoo.com.br

\section{MS. RODRIGO SANT'ANNA MARQUES}

Mestre em Educação Física pela Universidade Federal do Rio de Janeiro (UFRJ), Mestrando em Educação Física pelo Programa de Pós-graduação em Ciências do Esporte e do Exercício da Universidade Gama Filho (UGF) (Rio de Janeiro - Rio de Janeiro - Brasil)

E-mail:savio.vp@bol.com.br

\section{DRA. MONIQUE ASSIS}

Doutora em Educação Física pela Universidade Gama Filho (UGF), Docente do Programa de Pós-graduação em Ciências do Esporte e do Exercício da Universidade Gama Filho (UGF)

(Rio de Janeiro - Rio de Janeiro - Brasil)

E-mail:monique_assis@uol.com.br

\section{DRA. NILDA TEVES}

Doutora em Filosofia da Educação pela Universidade Federal do Rio de Janeiro (UFRJ), Docente do Programa de Pós-graduação em Ciências do Esporte e do Exercício da Universidade Gama Filho (UGF) (Rio de Janeiro - Rio de Janeiro - Brasil)

E-mail: tevesnilda@uol.com.br

I. Os autores do presente artigo declaram não possuir interesses pessoais, comerciais, acadêmicos, políticos ou financeiros nesse manuscrito. Declaramos, ainda, que o primeiro autor recebe auxílio do CNPq. 


\section{MS. JÉSSICA PRONESTINO DE LIMA MOREIRA}

Mestre em Saúde Coletiva pela Universidade Federal do Rio de Janeiro (UFRJ), Pesquisadora do Instituto de Estudos em Saúde Coletiva da Universidade Federal do Rio de Janeiro (IESC/NFRJ)

(Rio de Janeiro - Rio de Janeiro - Brasil)

E-mail: jessica@eefd.ufrj.br

\section{RESUMO}

O objetivo do presente estudo foi verificar a prevalência de insatisfação corporal entre adolescentes do sexo feminino na cidade do Rio de Janeiro. Foram investigadas 2. 149 estudantes do sexo feminino, entre 14 e 18 anos de idade. Foram aferidos os pesos, estaturas e índices de massa corporal (IMC) informados e desejados. A insatisfação corporal foi considerada de dois modos: a) através do desejo de modificar o IMC; e, b) o desejo de alterar o peso corporal em valor superior a 10\% do peso informado. Do total investigado, 93,58\% expressaram o desejo de modificar o IMC. Foi possível observar, ainda, que 42,48\% manifestaram insatisfação com o peso. É possível concluir, então, que as adolescentes investigadas manifestaram tendência à insatisfação com o peso corporal.

PALAVRAS-CHAVE: Imagem corporal; adolescentes; índice de massa corporal; peso corporal.

\section{INTRODUÇÃO}

Diferentes sociedades contemporâneas têm estimulado a magreza como um padrão de beleza e de saúde para o corpo, especialmente entre as mulheres. Os discursos que ecoam em campanhas publicitárias, revistas e programas televisivos parecem desencadear um poderoso conjunto de imperativos para determinar o modo como as pessoas devem conduzir suas próprias vidas e construir suas identidades. O sucesso e uma espécie de capital social têm sido associados, entre outros aspectos, à aparência física. Porém, estes padrões preestabelecidos podem suscitar sentimentos de inadequações e insatisfações com o corpo (NEIGHBORS; SOBAL, 2007; PHILIPS; DRUMMOND, 200 I; WRIGHT; O'FLYNN; MacDONALD, 2006; DiGIOACCHINO; SARGENT; TOPPING, 200I).

De uma forma geral, é mais comum que as pessoas do sexo feminino respondam com mais entusiasmo às mensagens socioculturais de atratividade corporal e assim, experimentam, com mais frequência, maior quadro de insatisfação com o corpo (NEIGHBORS; SOBAL, 2007; PHILIPS; DRUMMOND, 200 I).

A insatisfação com o próprio corpo está intimamente associada à prática de dietas e diferentes outras estratégias restritivas que, consequentemente, podem desencadear quadros clínicos de transtornos alimentares (BEARMAN et al., 2006; FURNHAM; BADMIN; SNEADE, 2002). Esta insatisfação com a imagem corporal é caracterizada pela percepção imprecisa, que resulta em uma ideia subestimada ou 
superestimada do tamanho de seu próprio corpo (McCABE et al., 2006). Assim, embora tenha sido comum estudar a insatisfação com o próprio corpo em populações que têm clinicamente diagnosticado transtornos alimentares, é crescente o interesse em investigar a insatisfação entre indivíduos que não apresentam tais distúrbios.

A pressão exercida sobre as mulheres para que tenham um corpo magro e longilíneo pode ter origem em diferentes setores da sociedade, como a mídia e a própria família (STICE; WHITENTON, 2002). Em adolescentes, esta pressão pode ser um importante fator para o desenvolvimento de insatisfação corporal, uma vez que a adolescência é um momento em que o indivíduo busca sua identidade pessoal e social, além de estar sofrendo mudanças corporais relacionadas à maturação (KRAYER; INGLEDEW; IPHOFEN, 2008).

Diversas pesquisas realizadas no Brasil têm indicado uma elevada prevalência de insatisfação corporal em distintas amostras (PERINI et al., 2009; COQUEIRO et al., 2008; PINHEIRO; GIUGLIANI, 2006; DAMASCENO et al., 2005). Um dos aspectos que merece destaque é o medo ou pavor de engordar, reportado por adolescentes do sexo feminino (PERINI et al., 2009; OLIVEIRA et al., 2003), já que pode indicar predisposição desses indivíduos à insatisfação com a imagem corporal e possivelmente aos transtornos do comportamento alimentar, em razão da preocupação constante com o controle do peso corporal demonstrado por essa conduta. Deste modo, estudos desta natureza poderiam contribuir para identificar, prevenir e auxiliar o tratamento de pessoas com problemas relacionados à imagem corporal.

Assim sendo, o objetivo do presente estudo foi verificar a prevalência de insatisfação com o corpo entre adolescentes do sexo feminino residentes na cidade do Rio de Janeiro. Especificamente, foram abordados: a) o desejo de ganhar, manter ou perder peso entre as adolescentes; e, b) alguns fatores associados à insatisfação.

\section{MÉTODO}

\section{AMOSTRA}

A população compreendeu todas as adolescentes do sexo feminino na faixa de 14 a 18 anos de idade, estudantes dos ensinos fundamental e médio em escolas públicas localizadas no município do Rio de Janeiro no ano de 2009, e residentes na mesma cidade. $O$ valor final estimado decorreu do número total de alunas do ensino fundamental e médio matriculadas nas escolas públicas localizadas nesta cidade durante o mesmo ano ( $\mathrm{N}=$ 105.000).

Para um erro amostral estipulado em 3\%, com nível de confiança de $99 \%$ e prevalência presumida de $50 \%$ de insatisfação com o corpo, foi estimado o tamanho 
amostral de 1.812 adolescentes. Precavendo-se de possíveis perdas, procurou-se levantar um número 20\% maior (2.174). Ao final, atingiu-se um total de 2.I49 adolescentes.

A seleção da amostra compreendeu, ainda, a escolha, por sorteio, das escolas públicas localizadas em diferentes regiões da cidade. No total, foram visitadas quarenta e sete escolas e nestas foram escolhidas as turmas que comportavam o maior número de alunas na faixa etária de interesse. Todas as alunas das turmas selecionadas foram convidadas a responderem o questionário. Foram adotados como critérios de exclusão: a) o fato de fazer I 4 anos no ano da coleta; b) estar grávida; c) ser portadora de deficiências físicas e/ou visuais; d) não residir na cidade; e, e) ter diagnosticado algum transtorno alimentar.

\section{INSTRUMENTOS}

\section{PESO, ESTATURA E ÍNDICE DE MASSA CORPORAL (IMC) INFORMADOS E DESEJADOS}

As informantes reportaram seus pesos e estaturas atuais e desejados, os quais permitiram que fosse calculado o IMC a partir da razão do peso sobre a estatura ao quadrado $\left(\mathrm{IMC}=\right.$ Peso $[\mathrm{kg}] /$ Estatura $\left.[\mathrm{m}]^{2}\right)$. Para efeito de análise, no presente estudo, estes dados foram denominados de peso, estatura e IMC "informado" ou "desejado". Os valores de corte para definir a classificação do IMC adotados foram: "abaixo do normal" ou "baixo peso" (IMC < I8,5); "normal" (I8,5 $\leq$ IMC $<25)$; e, "acima do normal" ou "sobrepeso/obesidade" ( $25 \leq$ IMC) (NEIGHBORS; SOBAL, 2007).

\section{INSATISFAÇÃO CORPORAL}

A insatisfação corporal foi considerada de dois modos distintos. Inicialmente foi avaliada através do desejo de diminuir ou aumentar a massa corporal. Para isso, foram utilizados o IMC informado e o IMC desejado. Quando a diferença entre os valores de IMC informado e desejado diferiu de zero, foi considerado que havia um quadro de insatisfação corporal (NEIGHBORS; SOBAL, 2007).

Por outro modo, foi analisado também o desejo de perder ou ganhar peso (em kg). Entretanto, neste caso, considerou-se que houve insatisfação corporal quando a diferença entre o peso informado e o peso desejado superou em I0\% o peso corporal informado. $O$ valor de corte de $10 \%$ foi escolhido por ser este o montante que pode ser, de forma realista, reduzido em programas de perda de peso bem desenhados (MAYNARD et al., 2006). 


\section{CLASSE SOCIAL}

Com o intuito de obter maiores informações socioeconômicas e culturais a respeito dos sujeitos investigados, aplicou-se o questionário sobre classe social da ABEP (Associação Brasileira de Empresas de Pesquisa) (SIQUEIRA et al., 2007).

\section{OUTROS DADOS DEMOGRÁFICOS}

Foram levantados, ainda, alguns dados demográficos como idade e bairro em que mora. Para efeito das análises, em relação ao bairro que residia, a amostra foi dividida em dois grupos. Um grupo comportou as residentes em bairros litorâneos, em que a praia faz parte do cotidiano dos moradores. $\bigcirc$ outro foi agrupado por adolescentes que moravam em bairros que não tinham praia.

\section{PROCEDIMENTOS}

Os procedimentos para a coleta dos dados foram: a) contato com as Secretarias Municipal e Estadual de Educação, encaminhamento do projeto e da carta de apresentação da pesquisa sob a égide da Universidade Federal do Rio de Janeiro, a fim de conseguir a aprovação para prosseguimento da investigação; b) seleção e visita às escolas mediante apresentação de autorização fornecida pelas Secretarias; c) seleção da(s) turma(s); d) envio do termo de consentimento para aprovação pelos estudantes e responsáveis; e) aplicação dos questionários às alunas.

\section{COMITÊ DE ÉTICA}

O presente estudo foi encaminhado e aprovado pelo Comitê de Ética e Pesquisa do Hospital Universitário Clementino Fraga Filho da Universidade Federal do Rio de Janeiro sob o número de protocolo 095/09.

\section{TRATAMENTO ESTATÍSTICO}

O tratamento estatístico utilizado estava de acordo com a natureza das variáveis. Foi realizada a frequência relativa de cada caso. A normalidade da distribuição amostral foi determinada pelo teste de Kolmogorov-Smirnov. $O$ teste t pareado foi utilizado para identificar se duas médias (de peso, estatura ou IMC informados e desejados) da amostra diferiram entre si. Para examinar as diferenças nas proporções das variáveis categóricas foi utilizado o Qui-quadrado. Para avaliar as diferenças entre duas ou mais categorias de ocorrência e o desejo de ganhar, manter ou perder massa 
ou peso foi usado o modelo logístico multinomial univariado. Foi utilizado, ainda, um modelo logístico multinomial multivariado para identificar as possíveis interferências no desfecho. Adotou-se o nível de significância de $p<0,05$.

\section{RESULTADOS}

Para as análises considerando a insatisfação corporal a partir da diferença entre os valores de IMC informado e desejado, foi observado que é significativo o desejo de perder peso (FIGURA IA), aumentar a estatura (FIGURA IB) e reduzir o IMC (FIGURA I C). Além disto, do total de 2. 149 estudantes investigadas, 2.01 I (93,58\%) expressaram o desejo de modificar sua massa corporal, sendo 70,82\% ( $n=1.522)$ com o intuito de perder massa corporal (FIGURA ID). Entre as adolescentes que querem somente perder massa, o valor médio de perda desejada de IMC foi de 3,48 pontos, enquanto, entre as que aspiram exclusivamente ganhar, o valor médio foi de 1,76 pontos (dados não observados nas Figuras).

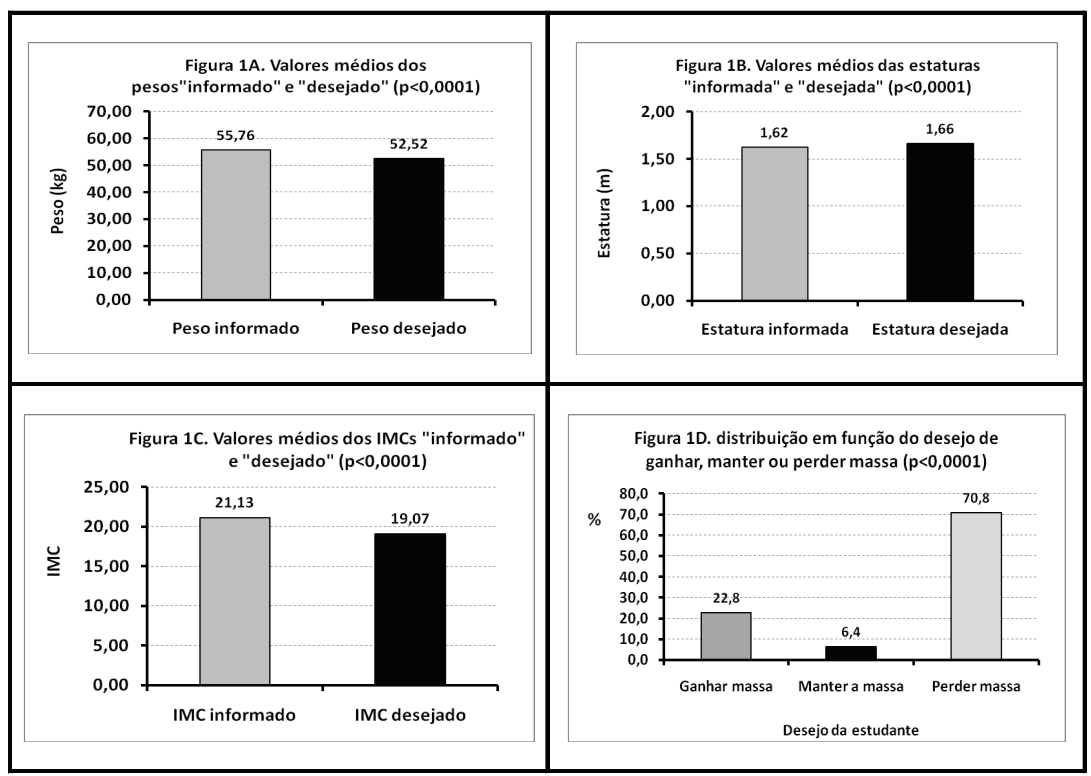

Figura I. Valores médios de peso, estatura, IMC e distribuição total da amostra em função do desejo de ganhar, perder ou manter a massa corporal.

As informantes com sobrepeso (IMC "acima do normal") demonstraram maior insatisfação com a massa corporal, quando comparadas às adolescentes "abaixo 
do normal" ou àquelas que apresentavam IMC aceito como "normal". Entretanto, entre as classificadas como "normal" ou "abaixo do normal", são poucas as que querem manter a massa corporal atual. As variáveis idade e classe social diferiram de modo significativo, de tal modo que adolescentes mais novas e de classe social mais elevada parecem apresentar maior insatisfação corporal. Por outro lado, a proximidade da residência à praia não parece influenciar na insatisfação com o próprio corpo (TABELA I).

Tabela I. Distribuição da amostra em função do desejo de ganhar, perder ou manter a massa corporal considerando as variáveis idade, classe social, bairro e o IMC atual

\begin{tabular}{|c|c|c|c|c|c|c|c|c|}
\hline & \multicolumn{6}{|c|}{ Desejo em relação à massa corporal } & \multirow{3}{*}{$\begin{array}{l}\text { p-valor } \\
\text { MLMU }\end{array}$} & \multirow{3}{*}{$\begin{array}{l}\text { p-valor } \\
\text { MLMM }\end{array}$} \\
\hline & \multicolumn{2}{|c|}{$\begin{array}{l}\text { Ganhar massa } \\
\text { corporal }\end{array}$} & \multicolumn{2}{|c|}{$\begin{array}{l}\text { Manter a massa } \\
\text { corporal }\end{array}$} & \multicolumn{2}{|c|}{$\begin{array}{l}\text { Perder massa } \\
\text { corporal }\end{array}$} & & \\
\hline & $\mathrm{n}$ & $\%$ & $\mathrm{n}$ & $\%$ & $\mathrm{n}$ & $\%$ & & \\
\hline \multicolumn{9}{|l|}{ Idades } \\
\hline 14 & 117 & 23,0 & 27 & 5,3 & 364 & 71,7 & & \\
\hline 15 & 115 & 20,1 & 31 & 5,4 & 425 & 74,4 & & \\
\hline 16 & 11 & 13,4 & 6 & 7,3 & 65 & 79,3 & $=0,012$ & $=0,000$ \\
\hline 17 & 149 & 23,4 & 44 & 6,9 & 445 & 69,7 & & \\
\hline 18 & 97 & 27,7 & 30 & 8,6 & 223 & 63,7 & & \\
\hline \multicolumn{9}{|l|}{ Classe social } \\
\hline$A$ & 8 & 18,6 & I & 2,3 & 34 & 79,1 & & \\
\hline B & 184 & 20,7 & 42 & 4,7 & 665 & 74,6 & & \\
\hline C & 274 & 23,9 & 91 & 7,9 & 783 & 68,2 & $=0,002$ & $=0,005$ \\
\hline$D+E$ & 23 & 34,3 & 4 & 6,0 & 40 & 59,7 & & \\
\hline \multicolumn{9}{|l|}{ Bairro que reside } \\
\hline Litorâneo & 31 & 16,6 & 13 & 7,0 & 143 & 76,5 & & \\
\hline Não litorâneo & 458 & 23,3 & 125 & 6,4 & 1379 & 70,3 & $=0,093$ & $=0,105$ \\
\hline \multicolumn{9}{|l|}{ IMC atual } \\
\hline Abaixo do normal & 280 & 58,0 & 36 & 7,5 & 167 & 34,6 & & \\
\hline Normal & 206 & 14,7 & $10 \mid$ & 7,2 & |09| & 78,0 & $=0,000$ & $=0,000$ \\
\hline Acima do normal & 3 & 1,1 & 1 & 0,4 & 264 & 98,5 & & \\
\hline
\end{tabular}

Legenda: MLMU (Modelo logístico multinomial univariado); MLMM (Modelo logístico multinomial multivariado).

Para as análises considerando a insatisfação como o desejo de perder ou ganhar peso em valores superiores a 10\% do peso atual, foi possível observar que 9। 3 (42,48\%) adolescentes manifestaram insatisfação com o corpo, sendo que 655 (30,45\%) desejariam perder peso, enquanto 258 (12,0 I \%) gostariam de ganhar 
peso. Em média, as adolescentes do primeiro grupo ambicionam perder I I,82kg e, as do segundo, aspiram ganhar 8,03kg. A Tabela 2 apresenta a associação de algumas variáveis com o desejo de ganhar, manter ou perder peso.

Tabela 2. Distribuição da amostra em função do desejo de ganhar, perder ou manter o peso considerando as variáveis idade, classe social, bairro e o IMC atual

\begin{tabular}{|c|c|c|c|c|c|c|c|c|}
\hline & \multicolumn{6}{|c|}{ Desejo em relação ao peso } & \multirow{3}{*}{$\begin{array}{l}p \text {-valor } \\
\text { MLMU }\end{array}$} & \multirow{3}{*}{$\begin{array}{l}p \text {-valor } \\
\text { MLMM }\end{array}$} \\
\hline & \multicolumn{2}{|c|}{ Ganhar peso } & \multicolumn{2}{|c|}{ Manter o peso } & \multicolumn{2}{|c|}{ Perder peso } & & \\
\hline & $\mathrm{n}$ & $\%$ & $\mathrm{n}$ & $\%$ & $\mathrm{n}$ & $\%$ & & \\
\hline \multicolumn{9}{|l|}{ Idades } \\
\hline 14 & 60 & 11,8 & 276 & 54,3 & 172 & 33,9 & \multirow{5}{*}{$=0,000$} & \multirow{5}{*}{$=0,000$} \\
\hline 15 & 65 & II,4 & 304 & 53,2 & 202 & 35,4 & & \\
\hline 16 & 6 & 7,3 & 47 & 57,3 & 29 & 35,4 & & \\
\hline 17 & 71 & 11,1 & 393 & 61,6 & 174 & 27,3 & & \\
\hline 18 & 56 & 16,0 & 216 & 61,7 & 78 & 22,3 & & \\
\hline \multicolumn{9}{|l|}{ Classe social } \\
\hline A & I & 2,3 & 32 & 74,4 & 10 & 23,3 & \multirow{4}{*}{$=0,032$} & \multirow{4}{*}{$=0,042$} \\
\hline B & 93 & 10,4 & 527 & 59,1 & 271 & 30,4 & & \\
\hline C & 153 & 13,3 & 639 & 55,7 & 356 & 31,0 & & \\
\hline$D+E$ & II & 16,4 & 38 & 56,7 & 18 & 26,9 & & \\
\hline \multicolumn{9}{|l|}{ Bairro que reside } \\
\hline Litorâneo & 17 & 9,1 & 120 & 64,2 & 50 & 26,7 & \multirow{3}{*}{$=0,130$} & \multirow{3}{*}{0,454} \\
\hline Não litorâneo & 241 & 12,3 & 1116 & 56,9 & 605 & 30,8 & & \\
\hline \multicolumn{7}{|l|}{ IMC atual } & & \\
\hline Abaixo do normal & 172 & 35,6 & 284 & 58,8 & 27 & 5,6 & \multirow{3}{*}{$=0,000$} & \multirow{3}{*}{$=0,000$} \\
\hline Normal & 85 & 6,1 & 921 & 65,9 & 392 & 28,0 & & \\
\hline Acima do normal & । & 0,4 & 31 & 11,6 & 236 & 88,1 & & \\
\hline
\end{tabular}

Legenda: MLMU (Modelo logístico multinomial univariado); MLMM (Modelo logístico multinomial multivariado).

\section{DISCUSSÃO}

No presente estudo, a prevalência de insatisfação corporal foi de 93,58\% quando verificado o desejo de alterar, em qualquer quantidade, a massa corporal; e de $42,48 \%$ quando observada a vontade de modificar o peso corporal em valores superiores a $10 \%$ do peso atual. Para a primeira forma de análise foi encontrado que $70,82 \%$ das adolescentes desejavam perder massa corporal, enquanto 30,45\% aspiravam perder peso, considerando o segundo modo de análise. Estes valores parecem não diferir muito dos resultados encontrados em outras investigações. 
Neighbors e Sobal (2007) observaram que 90,3\% das estudantes universitárias norteamericanas (idade média $=20,0$ anos) que fizeram parte da amostra manifestaram desejo de alterar o peso corporal e $84,4 \%$ ambicionaram perder peso. Hayashi et al. (2006) verificaram que 53,5\% das moças entre I 5 e 19 anos de idade de diferentes regiões no Japão desejavam ser magras, enquanto Suka et al. (2006), também no Japão, mas com crianças de 12 e 13 anos de idade, encontraram que $42,0 \%$ gostariam de ser mais magras. Em pesquisa com adolescentes do sexo feminino do ensino fundamental e médio, entre 10 e 19 anos de idade, da cidade de Florianópolis, Alves et al. (2008) identificaram que 18,8\% manifestaram insatisfação corporal, embora nesta pesquisa os autores tenham utilizado outro instrumento para detecção da insatisfação corporal.

Através dos dados obtidos no presente estudo, em qualquer que fosse o tipo de análise, observou-se um desejo maior em perder do que ganhar massa ou peso corporal. Esta tendência é averiguada também em outros estudos (NEIGHBORS; SOBAL, 2007; HAYASHI et al., 2006; SUKA et al., 2006; DiGIOACCHINO; SARGENT; TOPPING, 200 I ). Assim, embora tenhamos verificado a diferença de 2,06 pontos entre os IMCs "informado" e "desejado", encontramos, em nossa pesquisa, entre as adolescentes que querem perder massa, o desejo de perder 3,48 pontos no IMC, enquanto Neighbors e Sobal (2007) encontraram, em média, o desejo de reduzir 1,4 pontos entre universitárias norteamericanas e DiGioacchino, Sargent e Topping (200I ) 2,32 pontos em estudantes universitárias norteamericanas de pele branca e 2, 10 pontos nas de pele negra. É preciso ressaltar, porém, que os estudos de Neighbors e Sobal (2007) e de DiGioacchino, Sargent e Topping (200 I) foram realizados com estudantes ligeiramente mais velhas (aproximadamente 20 anos de idade, em média). Por outro lado, Neighbors e Sobal (2007) observaram que 4\% das estudantes investigadas com o IMC classificado como "normal" gostariam de aumentar o peso. Este desejo aumentava consideravelmente (50\%), contudo, quando as estudantes eram classificadas como "magras" pelo IMC. Em nosso estudo, verificamos o desejo de $22,76 \%$ das estudantes ganharem massa e 12,03\% aumentarem o peso, dependendo do tipo de análise.

Os resultados da presente análise demonstraram que as idades de 15 e 16 anos foram mais problemáticas em relação à vontade de perder massa ou peso corporal, enquanto o desejo de ganhar massa ou peso parece ocorrer mais tardiamente, aos 18 anos de idade. Al Sabbah et al. (2009) encontraram associação positiva entre a insatisfação com o peso corporal e a idade ao pesquisarem adolescentes de II a I 5 anos de idade. Bearman et al. (2006), ao analisarem a insatisfação com a imagem corporal em adolescentes de 12 a 16 anos, verificaram que a idade é um preditor para a insatisfação corporal, com as meninas mais velhas apresentando 
maior insatisfação com seus corpos quando comparadas às mais jovens. Desfecho semelhante é observado no estudo de Zaborskis et al. (2008) que, ao verificarem a insatisfação corporal em adolescentes de 13 e 15 anos de idade, também encontraram maior prevalência desta variável entre as meninas mais velhas - 41, 1\% das meninas com 15 anos de idade eram insatisfeitas com seus corpos, contra 32,7\% das meninas com 13 anos de idade.

Verificamos, também, que a perda de massa corporal ramosfoi influenciada pela classe social. Em nossa análise, considerando qualquer valor da diferença entre o IMC informado e o desejado, observamos que foi na classe mais elevada que houve o maior desejo de perder massa corporal. Por outro lado, a classe mais alta foi a que teve menor desejo de reduzir o peso, quando consideradas somente aquelas que desejavam alterar em mais de $10 \%$ o peso corporal. Interessante notar que, apesar de ser um fator frequentemente associado à proteção de diversas enfermidades (ERGINOZ et al., 2004), a elevada condição socioeconômica é apontada como um fator de risco para a insatisfação corporal em adolescentes (O'DEA, 2008). Segundo a autora, sujeitos pertencentes às classes socioeconômicas mais abastadas seriam mais propensos a apresentarem maior insatisfação corporal do que aqueles que compõem os estratos socioeconômicos menos favorecidos, hipótese esta fomentada pelos dados de diversos estudos (CEBALLOS; CZYEWSKA, 20 I0; LYNCH et al., 2007; WANG et al., 2005; GLUCK; GELIEBTER, 2002).

Na presente investigação não foram encontradas diferenças significativas entre o desejo de perder massa ou peso corporal e a localidade em que os sujeitos residiam. Parece que o fato de morar próximo à praia não influenciou a insatisfação corporal. Em estudo realizado no Canadá, McLaren e Gauvin (2003) não encontraram variação na insatisfação corporal entre vizinhanças pesquisadas pertencentes a diferentes estratos socioeconômicos.

A cidade do Rio de Janeiro, como ressalta Farias (2002), exibe suas praias como uma insígnia de sua maneira de ser, sua identidade. Segundo a autora, não é surpreendente que os bairros Copacabana e Ipanema, com suas praias famosas, representem bem a cidade. Neste sentido, a ostentação do corpo seminu nesses locais pode estar relacionada a sentimentos de autossatisfação, e é possível supor que a região litorânea possa ter influência sobre a insatisfação corporal. Contudo, a antropóloga, em pesquisa no posto nove, típica localidade da praia de Ipanema, não verificou este fato, o que corrobora os dados observados no presente estudo.

Cabe destacar que a insatisfação com a imagem corporal tem sido aceita como um importante fator de risco para a manifestação de anorexia nervosa e deveria receber maior atenção daqueles que lidam com adolescentes (ALVES, 2008). 
propósito do estudo foi verificar a prevalência de insatisfação corporal entre adolescentes do sexo feminino; identificar o desejo de ganhar, manter ou perder peso e examinar alguns fatores associados à insatisfação. $\bigcirc$ estudo permitiu concluir, então, que há uma expressiva insatisfação com o próprio corpo entre as adolescentes do sexo feminino residentes na cidade do Rio de Janeiro. A partir das análises do IMC, 93,58\% expressaram o desejo de modificar sua massa corporal. De outra forma, considerando-se a variação de $10 \%$ do peso corporal, a taxa de prevalência de insatisfação situou-se em 42,48\%. Nos dois casos houve um predominante desejo de perder massa ou peso corporal (70,82\% para a primeira análise e 30,45\% para a segunda), evidenciando uma característica associada à magreza, tão difundida, atualmente, nos veículos de comunicação. Entre os fatores investigados, associaram-se significativamente à insatisfação corporal, independente da forma de análise, a idade entre I 5 e 16 anos, a classe social mais elevada e o IMC informado elevado. Tal fato é de extrema relevância na medida em que a insatisfação corporal é um importante fator de risco para os transtornos alimentares, tais como a anorexia e a bulimia.

Por outro lado, mais pesquisas são necessárias para que se possa compreender com maior profundidade os fatores associados à insatisfação corporal, bem como sua prevalência em diferentes cidades brasileiras. De todo modo, talvez seja possível sugerir que o debate sobre a insatisfação corporal encontre reflexão nas escolas, uma vez que tem ganhado importância, entre os jovens da sociedade contemporânea, este tipo de preocupação.

Dissatisfaction with mass and body weight among Elementary and High School female students in the city of Rio de Janeiro

ABSTRACT: The aim of this study was to verify the prevalence of body dissatisfaction among adolescent girls in the city of Rio de Janeiro. 2, 149 female students aged between 14 and 18 years were investigated. Informed and desired weights, heights and the body mass index (BMI) were also investigated. The body dissatisfaction was addressed in two ways: a) by the desire to change the $\mathrm{BMI}$ and b) the desire to change the weight (in $\mathrm{kg}$ ) over 10\% of body informed. Of the total, 93.58\% expressed a desire to modify their BMI. We also observed that $42.48 \%$ reported dissatisfaction with the weight. Therefore, it was possible to conclude that female students showed a tendency to be dissatisfied with their body weight. KEYWORDS: Body image; adolescents; body mass index; body weight. 
Insatisfacción com el peso y masa corporal entre los Estudiantes de la escuela primaria y secundaria del sexo femenino em la ciudad de Rio de Janeiro

RESUMEN: El objetivo del presente estudio fue determinar la prevalencia de la insatisfacción corporal en adolescentes del sexo femenino en la ciudad de Río de Janeiro. Se investigaron 2. 149 estudiantes con edades comprendidas entre 14 y 18 años. Se levantaron los pesos, las alturas y los índices de masa corporal (IMC) informados y deseados. La insatisfacción con el cuerpo fue considerada de dos maneras: a) por el deseo de cambiar el IMC y b) el deseo de cambiar el peso (en kg) con valores superiores a los 10\% del peso informado. Del total, 93,58\% expresó el deseo de cambiar su IMC. También observamos que 42,48\% reportaron insatisfacción con el peso. Es posible concluir que las adolescentes encuestadas expresaron una tendencia de insatisfacción con su peso corporal.

PALABRAS CLAVE: Imagen corporal; adolescentes; índice de masa corporal; peso corporal.

\section{REFERÊNCIAS}

ALVES, E. et al. Prevalência de sintomas de anorexia nervosa e insatisfação com a imagem corporal em adolescentes do sexo feminino do Município de Florianópolis, Santa Catarina, Brasil. Cadernos de Saúde Pública, Rio de Janeiro, v. 24, n. 3, p. 503-5I2, mar. 2008.

AL SABBAH, H. et al. Body weight dissatisfaction and communication with parents among adolescents in 24 countries: international cross-sectional survey. BMC Public Health, London, v. 9, n. 52, feb. 2009.

BEARMAN, S. K. et al. The skinny on body dissatisfaction: a longitudinal study of adolescent boys and girls. Journal of Youth and Adolescence, New York, v. 35, n. 2, p. 217-229, apr. 2006.

CEBALLOS, N.; CZYEWSKA, M. Body image in Hispanic/Latino vs. European American adolescents: implications for treatment and prevention of obesity in underserved populations. Journal of Health Care for the Poor and Underserved, Nashville, v. 21, n. 3, p. 823-838, aug. 2010.

COQUEIRO, R. S. et al. Insatisfação com a imagem corporal: avaliação comparativa da associação com estado nutricional em universitários. Revista de Psiquiatria do Rio Grande do Sul, Porto Alegre, v. 30, n. I, p. 31-38, jan/abr. 2008.

DAMASCENO, V.O. et al. Tipo físico ideal e satisfação com a imagem corporal de praticantes de caminhada. Revista Brasileira de Medicina do Esporte, São Paulo, v. I I, n.3, p. |8|-186, mai.jun. 2005.

DiGIOACCHINO, R. F; SARGENT, R. G.; TOPPING, M. Body dissatisfaction among white and african american male and female college students. Eating Behaviors, New York, v. 2, n. I, p. 39-50, spr. 2001 .

ERGINOZ, E. et al. Perceived health status in a Turkish adolescent sample: risk and protective factors. European Journal of Pediatrics, Berlin, v. 163, n. 8, p. 485-494, aug. 2004. 
FARIAS, P. Corpo e classificação de cor numa praia carioca. In: GOLDENBERG, M. (Org.). Nu \& vestido: dez antropólogos revelam a cultura do corpo carioca. Rio de Janeiro, 2002: Record, 2002. p. 263-302.

FURNHAM, A.; BADMIN, N.; SNEADE, I. Body image dissatisfaction: gender differences in eating attitudes, self-esteem, and reasons for exercise. The Journal of Psychology, Provincetown, v. 136, n. 6, p. 581-596, nov. 2002.

GLUCK, M. E.; GELIEBTER, A. Racial/ethnic differences in body image and eating behaviors. Eating Behaviors, New York, v. 3, n. 2, p. I43-I5I, sum. 2002.

HAYASHI, F. et al. Perceived body size and desired for thinnes of young Japanese women: a population-based survey. British Journal of Nutrition, Cambridge, v. 96, n. 6, p. I I 54- | |62, dec. 2006.

KRAYER, A.; INGLEDEW, D. K.; IPHOFEN, R. Social comparison and body image in adolescence: a grounded theory approach. Health Education Research, Oxford, v. 23, n. 5, p. 892-903, oct. 2008.

LYNCH, E. et al. Association of ethnicity and socioeconomic status with judgments of body size: the coronary artery risk development in young adults (CARDIA) study. American Journal of Epidemiology, Baltimore, v. 165, n. 9, p. 1055-1062, may 2007.

MCCABE, M. P. et al. Accuracy of body size estimation: Role of biopsychosocial variables. Body Image, Amsterdam, v. 3, n. 2. p. 163-171, jun. 2006.

MCLAREN, L.; GAUVIN, L. Does the 'average size' of women in the neighbourhood influence a woman's likelihood of body dissatisfaction? Health \& Place, Kidlington, v. 9, n. 4, p. 327-335, dec. 2003.

MAYNARD, L. M. et al. Secular trends in desired weight of adults. International Journal of Obesity, London, v. 30, p. I375- I38I, sep. 2006. NEIGHBORS, L. A.; SOBAL, J. Prevalence and magnitude of body weight and shape dissatisfaction among university students. Eating Behaviors, New York, v. 8, n. 4, p. 429-439, dec. 2007.

O'DEA, J. A. Gender, ethnicity, culture and social class influences on childhood obesity among Australian schoolchildren: implications for treatment, prevention and community education. Health and Social Care in the Community, Oxford, v. 16, n. 3, p. 282-290, may 2008.

OLIVEIRA, F. P. et al. Comportamento alimentar e imagem corporal em atletas. Revista Brasileira de Medicina do Esporte, São Paulo, v. 9, n. 6, p. 348-356, nov./dez. 2003.

PERINI, T. A. et al. Transtorno do comportamento alimentar em atletas de elite de nado sincronizado. Revista Brasileira de Medicina do Esporte, São Paulo, v. I 5, n. I, p. 54-57, jan./ fev. 2009. 
PHILIPS, J.M.; DRUMMOND, M.J.N. An investigation into the body image perception, body satisfaction and exercise expectations of male fitness leaders: implications for professional practice. Leisure Studies, Abingdon, v. 20, p. 95-105, apr. 2001.

PINHEIRO, A. P.; GIUGLIANI, E. R. J. Body dissatisfaction in Brazilian schoolchildren: prevalence and associated factors. Revista de Saúde Pública, São Paulo, v. 40, n. 3, p. 489-496, jun. 2006.

SIQUEIRA, F. V. et al. Prevalência de quedas em idosos e fatores associados. Revista de Saúde Pública, São Paulo, v. 4I, n. 5, p. 749-56, out. 2007.

STICE, E.; WHITENTON, K. Risk factors for body dissatisfaction in adolescent girls: a longitudinal investigation. Developmental Psychology, Richmond, v. 38, n. 5, p. 669-678, sept. 2002.

SUKA, M. et al. Body image, body satisfaction and dieting behavior in Japanese preadolescents: The Toyama Brith Cohort Study. Environmental Health and Preventive Medicine, Sapporo, v. I I, n. I, p. 24-30, jan. 2006.

WANG, Z. et al. Influences of ethnicity and socioeconomic status on the body dissatisfaction and eating behavior of Australian children and adolescents. Eating Behaviors, New York, v. 6, n. I, p. 23-33, jan. 2005.

WRIGHT, J.; O'FLYNN, G.; MACDONALD, D. Being Fit and Looking Healthy: Young Women's and Men's Constructions of Health and Fitness. Sex Roles, Pittsburgh, v. 54, n. 9-10, p. 707-7I6, may. 2006.

ZABORSKIS, A. et al. Body image and weight control among adolescents in Lithuania, Croatia, and the United States in the context of global obesity. Croatian Medical Journal, Zagreb, v. 49, n. 2, p. 233-242, apr. 2008.

Recebido em: 3 ago. 201 I Aprovado em: 24 maio 2012

Endereço para correspondência: Alexandre Palma

Universidade Federal do Rio de Janeiro Av. Carlos Chagas Filho, 540, Edifício da Educação Física Cidade Universitária Rio de Janeiro, RJ, Brasil CEP: 2194I-599 